\title{
Nonlinear Distortions and Parametric Amplification Generate Otoacoustic Emissions and Increased Hearing Sensitivity
}

\author{
Frank Böhnke $\mathbb{D}$ \\ Department of Otorhinolaryngology, Klinikum rechts der Isar, Technical University of Munich, \\ Ismaningerstr. 22, 81675 München, Germany; frank.boehnke@tum.de
}

Received: 29 May 2019; Accepted: 23 July 2019; Published: 2 August 2019

\begin{abstract}
The ear is able to detect low-level acoustic signals by a highly specialized system including a parametric amplifier in the cochlea. This is verified by a numerical mechanical model of the cochlea, which reduces the three-dimensional (3D) system to a one-dimensional (1D) approach. A formerly developed mechanical model permits the consideration of the fluid and the orthotropic basilar membrane in a 1D fluid-structure coupled system. This model shows the characteristic frequency to place transformation of the traveling wave in the cochlea. The additional inclusion of time and space dependent stiffness of outer hair cells and the signal level dependent stiffness of the string enables parametric amplification of the input signal. Due to the nonlinear outer hair cell stiffness change, nonlinear distortions follow as a byproduct of the parametric amplification at low levels constituting the compressive nonlinearity. More distortions are generated by the saturating displacements of the string at high input levels, which can be distinguished from the low-level distortions by the order of additional harmonics. Amplification factors of $15.5 \mathrm{~dB}$ and $24.0 \mathrm{~dB}$ are calculated, and a change of the traveling-wave mapping is postulated with parametric amplification representing the healthy state of the cochlea.
\end{abstract}

Keywords: Cochlea; Parametric amplification; Outer hair cells; Nonlinear distortions

\section{Introduction}

The hearing system, including the cochlea of the inner ear, has the ability to detect low level acoustic signals in the subnanometer range to enable efficient and sensitive communication. This extraordinary feature is induced by a highly specialized mechano-electrical system transforming air-guided sound to vibrations along the basilar membrane (BM) surrounded by waterlike fluid. It is accepted that traveling waves in the mammalian cochlea show a frequency to place mapping, in the sense that the place of maximum displacement along the BM depends on the frequency of the stapes movement. This work confirms that statement and extends it by including small axial nonlinear stiffness changes of outer hair cells $(\mathrm{OHC})$, with the effect of parametric amplification and a place shift of maximum BM displacement during propagation of the traveling wave. The concept of parametric traveling-wave amplification was predicted considering strings [1] and electric network circuits [2]. Furthermore, it was shown in the theory of nonlinear oscillations that two harmonic forces with circular frequencies $\omega_{1}$ and $\omega_{2}$ will produce additional components which contain not only these circular frequencies but combination tones with multiple $n \omega_{1} \pm m \omega_{2}$ and also subharmonic frequencies [3,4].

The cochlear model presented is a 1D approach of the fluid-structure coupled mechanics formulated by a partial differential equation (PDE) in time and one-space dimension. The solution of the PDE is executed by a finite difference scheme including a loss term in a damped wave equation. Another nonlinear term, standing for the restoring force, which increases with the cube of 
displacements, becomes relevant for large displacements. Recently similar approaches for strings with viscoelastic foundations [5] and formerly with hydrodynamic bearings have been presented [6,7].

\section{Methods}

\subsection{Partial Differential Equation (Parametric Traveling-Wave Amplifier)}

To prove the effect of parametric amplification (PA) of acoustic signals for increasing the hearing sensitivity of the cochlea, we study a 1D cochlear mechanical model in the form of a partial differential equation based on a former passive approach [8]. The reduction of dimensionality from $3 \mathrm{D}$ to $1 \mathrm{D}$ or $2 \mathrm{D}$ involves various limitations. One of these is the impossibility to calculate sound wave reflections at rigid walls or walls having limited mechanical impedance values. Even more important is the inability to simulate the 3D field generated by the superposition of propagating sound waves in a complex fluid-filled geometry, like the cochlea, including the coupling to the elastic BM. An alternative hypothesis of a 2D approach relies on the same assumption of a 1D model concerning the structure (BM), namely, representing the orthotropic elastic shell (BM) as a 1D embedded elastic beam. Therefore, boundary layers and interactions of 3D sound fields cannot be calculated properly as well. The derivation of the PDE for the traveling-wave amplifier in the cochlea is based on a structural cochlear model which reduces the 3D geometry to a $1 \mathrm{D}$ approach, representing the $\mathrm{BM}$ as an elastic embedded beam [8] (Figures 1 and 2). The fluid structure coupling relies on the assumption that the $\mathrm{BM}$ displacement is equal to the fluid (volume) velocity gradient in the longitudinal direction of the cochlea. The 3D box-model of the uncoiled cochlea includes the fluid (perilymph) filled Scala vestibuli (S.v.) and Scala tympani (S.t.). The Scala media is disregarded in a first approach. For simplification, the bending stiffness of the resulting elastic embedded beam (Figure 2) is neglected and Equation (1) - similar to an equation describing the transverse displacements of a string - is developed. If the effects of the longitudinal BM coupling and the inertial forces caused by the low BM mass compared to the fluid mass are neglected, Equation (1) obtains the pressure results. The displacement $u=u(x, t)$ is a function of space and time.

$$
\rho_{f l} \frac{\partial^{2} u}{\partial t^{2}}+E_{y} h^{3} S \frac{\partial^{2}}{\partial x^{2}}\left[\frac{u}{b^{4}(x)}\right]=0
$$
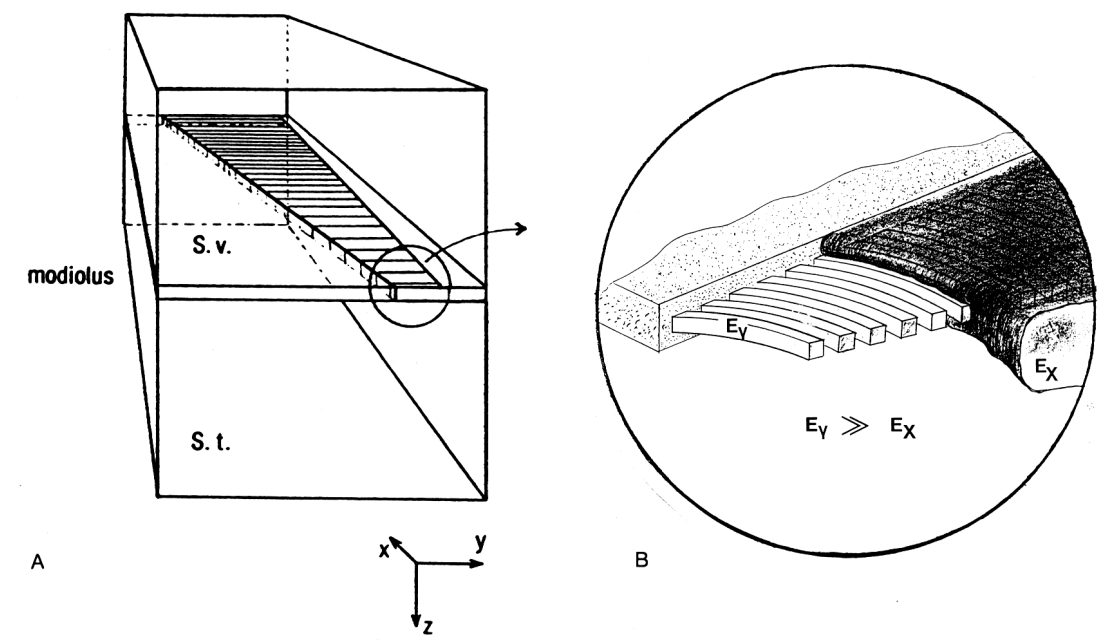

Figure 1. Box model of the human cochlea (A). The upper front area (representing the oval window attaching the Scala vestibuli (S.v.)) is excited by a sinusoidal velocity with the frequency $2 \mathrm{kHz}$ and amplitude $1 \mathrm{~nm} / \mathrm{s}$. The magnified view of the basilar membrane (BM) (B) illustrates the orthotropic consistency of the BM with collagen containing stiff transverse fibers with Young's modulus $E_{y}$ embedded into softer elastic tissue $E_{x}<<E_{y}$. 


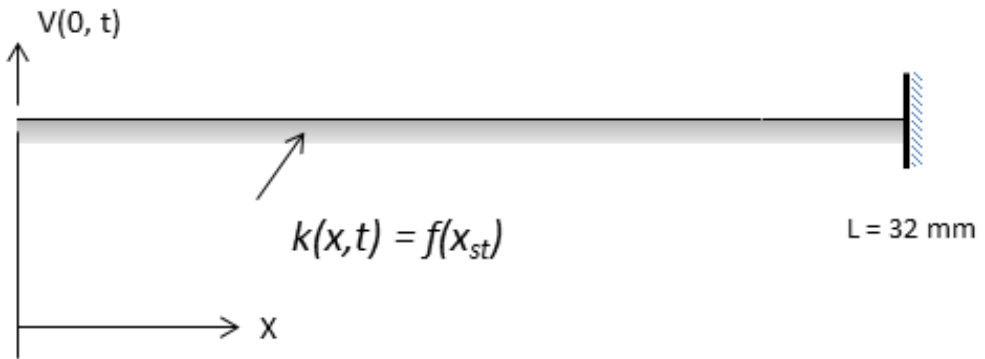

Figure 2. Elastic string representing the BM with nonlinear stiffness $k(x, t)$, produced by outer hair cells $(\mathrm{OHC})$ in the cochlea.

Though known physical damping mechanisms such as boundary layer damping $[9,10]$ are relevant in the cochlea, an overall standard viscous damping term is included in PDE (2). Furthermore, a time and space dependent Young's modulus change $\Delta E_{y}$, representing the stiffness change by $\mathrm{OHC}$ along the BM, is included in (2). With the so far mentioned physical portions, an unlimited increase of displacements would result with increasing input signal amplitude. A displacement limiting cubic nonlinear term $k_{c u b} u^{3}$, considering the stiffening of a string with increasing displacement amplitude, is added. The cubic term assures the increasing restoring force for positive and negative displacements of the string representing the BM. Therefore, the transversal displacements cannot grow beyond all limits as in the unphysiological linear case.

$$
\rho_{f l} \frac{\partial^{2} u}{\partial t^{2}}+k_{d} \frac{\partial u}{\partial t}+\left[E_{y}+\Delta E_{y}(x, t)\right] h^{3} S \frac{\partial^{2}}{\partial x^{2}}\left[\frac{u}{b^{4}(x)}\right]+k_{c u b} u^{3}=0 .
$$

The equations so far are noted in the time domain without the restriction of the stationarity of input signals. Multifrequency and transient stimulations, as used in otological diagnostics, can be utilized and associated BM displacements are calculated. Table 1 summarizes the geometric and physical parameters of the 1D cochlear model.

Table 1. Physical parameters constituting the 1D cochlear model with parametric amplification.

\begin{tabular}{lll}
\hline Symbol & Denotation & Value Unit \\
\hline$\rho_{f l}$ & fluid density per unit area & $1000 \mathrm{~kg} \mathrm{~m}^{-2}$ \\
$\mathrm{~b}(\mathrm{x})$ & BM width & $0.1-0.5 \mathrm{~mm}$ \\
$\mathrm{u}(\mathrm{x}, \mathrm{t})$ & BM displacement & $\mathrm{m}$ \\
$k_{d}$ & damping constant & $4000 \mathrm{~kg} \mathrm{~m}^{-2} \mathrm{~s}^{-1}$ \\
$k_{c u b}$ & cubic nonlinearity constant & $-10^{6} \mathrm{~kg} \mathrm{~m}^{-4} \mathrm{~s}^{-2}$ \\
$E_{y}$ & transverse Young's modulus & $100 \mathrm{GPa}$ \\
$\Delta E_{y}(x, t)$ & variable Young's modulus & $\mathrm{Pa}$ \\
$h$ & BM thickness & $10 \mu \mathrm{m}$ \\
$S$ & cross section area of canal & $1 \mathrm{~mm}$ \\
$L$ & BM length & $32 \mathrm{~mm}$ \\
\hline
\end{tabular}

Equation (2) is solved for the BM from $0 \mathrm{~mm}$ to $L=32 \mathrm{~mm}$. The width of the BM increases linearly from $b_{0}=0.1 \mathrm{~mm}$ at the base of the cochlea to $b_{L}=0.5 \mathrm{~mm}$ at the helicotrema. If a constant 
value $b(0)=0.1 \mathrm{~mm}$ is assumed, Equation (1) reduces to the standard wave equation with constant coefficients describing the propagation of displacements with the velocity

$$
v e l=\sqrt{\frac{E y \cdot h^{3} \cdot S}{\rho_{f} \cdot b_{0}^{4}}}=31.62 \mathrm{~ms}^{-1} .
$$

\subsection{Finite Difference Scheme (Damped Wave Equation)}

Equation (2) is solved by applying an explicit finite difference scheme (3) according to those presented in [11].

$$
u_{i}^{n+1}=c_{1}\left(2 u_{i}^{n}-u_{i}^{n-1}\right)+c_{2} u_{i}^{n-1}+\frac{v e l^{2}}{c_{3} \Delta x^{2}} \cdot\left(\frac{u_{i+1}^{n}}{\left(b / b_{0}\right)_{i+1}^{4}}-\frac{2 u_{i}^{n}}{\left(b / b_{0}\right)_{i}^{4}}+\frac{u_{i-1}^{n}}{\left(b / b_{0}\right)_{i-1}^{4}}\right)+k_{c u b}\left(u_{j}^{n}\right)^{3} .
$$

The $\left(b / b_{0}\right)^{4}$ terms are time independent but space variable-normed BM width factors. The normed damping constant of dimension $\mathrm{s}^{-1}$ is $k_{d}^{\prime}=k_{d} \cdot \mathrm{m}^{2} \mathrm{~kg}^{-1}$. Further abbreviations are

$$
c_{1}=\frac{\frac{1}{\Delta t^{2}}}{\frac{1}{\Delta t^{2}}+\frac{k_{d}^{\prime}}{2 \Delta t}}, \quad c_{2}=\frac{\frac{k_{d}^{\prime}}{2 \Delta t}}{\frac{1}{\Delta t^{2}}+\frac{k_{d}^{\prime}}{2 \Delta t}}, \quad c_{3}=\frac{1}{\Delta t^{2}}+\frac{k_{d}^{\prime}}{2 \Delta t^{\prime}}
$$

with $i, n$ denoting space and time indices, respectively. The resolution in space is $\Delta x=32 \mathrm{~mm} / 200=0.16 \mathrm{~mm}$; and in time $\Delta t=1 \mu \mathrm{s}$. All results are evaluated $100 \mathrm{~ms}$ after onset, according to $10^{5}$ time steps. The average calculation time is 30 minutes using a Z800 Workstation (Hewlett Packard, Palo Alto, CA, USA) equipped with 160 GByte RAM.

\subsection{Nonlinear Stiffness Function of Outer Hair Cells}

We assume that nonlinear stiffness changes of OHC cause time dependent variations of the Young's modulus $\Delta E_{y}(x, t)$ and the generation of nonlinear distortions and parametric amplification in the cochlea. For a derivation, we follow the assumption that the nonlinear BM motion is due to the nonlinear growth function of the OHC membrane potential [12]. The outer hair cell membrane potential $V_{m p}$ is fitted by the second-order Boltzmann function

$$
V_{m p}=V_{h}+\frac{V_{p p}}{1.0+e^{-z_{1}\left(x_{s t}-x_{1}\right) / k T} \cdot\left[1.0+e^{-z_{2}\left(x_{s t}-x_{2}\right) / k T}\right]} .
$$

$V_{p p}$ is the peak-to-peak value and $V_{h}$ is the membrane potential for maximum hyperpolarization. The constants $z_{1}$ and $z_{2}$, and displacements $x_{1}$ and $x_{2}$, are determined by the Levenberg-Marquardt algorithm, respectively, and $k T$ is the product of the Boltzmann-constant $k$ and the temperature $T$ (Table 2). In the next step, the somatic axial stiffness of an OHC as a function of the membrane potential is derived. This is impossible for the in vivo case, and therefore experimental results of isolated $\mathrm{OHCs}$ are used $[13,14]$. Applying again the Levenberg-Marquardt algorithm to fit experimental data of OHC stiffness measurements, a stiffness function is obtained

$$
k\left(x_{s t}\right)=k_{0}\left(1+a e^{b V_{m p}}+c e^{d V_{m p}}\right),
$$

with constants $a, b, c, d$, and $k_{0}$-the constant OHC stiffness is developed (Table 2). The nonlinear Equations (4) and (5) describe the axial somatic stiffness change in dependence of the $\mathrm{OHC}$ stereocilia bundle displacements $k(x, t)=f\left(x_{s t}(t)\right)$. The stiffness $k\left(x_{s t}\right)$ multiplied by a factor $f_{\text {act }}$ is inserted into Equation (3) and corresponds to a velocity change and Young's modulus change $\Delta E_{y}(x, t)$ in Equation (2). The factor $f_{\text {act }}$ was optimised by numerical experiments before an instability (unlimited growth) of displacements occured. The equivalent acoustic velocity change according to the nonlinear 
stiffness of $\mathrm{OHC}$ is $1.7 \mu \mathrm{m} \mathrm{s}^{-1}$, and therefore, a small amount $0.53 \times 10^{-9}$ of the undisturbed velocity which is vel $=31.62 \mathrm{~m} \mathrm{~s}^{-1}$.

Table 2. Electrical and mechanical parameters constituting the parametric amplification by outer hair cells.

\begin{tabular}{lll}
\hline Symbol & Denotation & Value Unit \\
\hline$V_{m p}$ & OHC membrane potential & $V_{h} \rightarrow-61 \mathrm{mV}$ \\
$V_{h}$ & OHC membrane potential for maximum hyperpolarization & $-65.9 \mathrm{mV}$ \\
$z_{1}, z_{2}$ & force factors & $60 \mathrm{fN}, 120 \mathrm{fN}$ \\
$x_{1}, x_{2}$ & displacements & $56.8 \mathrm{~nm}, 27.3 \mathrm{~nm}$ \\
$k T$ & Boltzmann-constant $\times$ temperature (energy) & $4.11 \times 10^{-21} \mathrm{~J}$ \\
$x_{s t}$ & stereocilia displacement & $\mathrm{m}$ \\
$k_{0}$ & OHC stiffness & $0.001 \mathrm{Nm}^{-1}$ \\
$a, b$ & constants & $0.001415,-0.0419$ \\
$c, d$ & constants & $2.665,-0.003801$ \\
$f_{a c t}$ & factor & $8.2 \times 10^{-10}$ \\
\hline
\end{tabular}

\section{Results}

The input signal is the time-dependent displacement $u(0, t)=\hat{u} \cdot \sin (\omega t)$ at $x=0 \mathrm{~mm}$, with $\hat{u}=1 \mathrm{~nm}$ representing the displacement amplitude of the oval window at the base of the cochlea. The solution of Equation (2) is evaluated by the finite-difference scheme (3). We study six cases and present four of them, which are collected in Table 3 using a switched sinusoidal signal of frequency $2 \mathrm{kHz}$ and two input amplitudes $1 \mathrm{~nm}$ and $100 \mathrm{~nm}$, respectively.

Table 3. Passive and active cases with $40 \mathrm{~dB}$ input level difference.

\begin{tabular}{llll}
\hline Input Amplitude & Linear (Passive) & Cubic Distortion (Passive) & OHC Nonlinear Stiffness (Active) \\
\hline $1 \mathrm{~nm}$ & Figure 2 space & - & Figure 3 space \\
& Figure 4 spectrum basal & - & Figure 5 spectrum medial \\
$100 \mathrm{~nm}$ & - & Figure 6 spectrum basal & Figure 7 spectrum medial \\
\hline
\end{tabular}

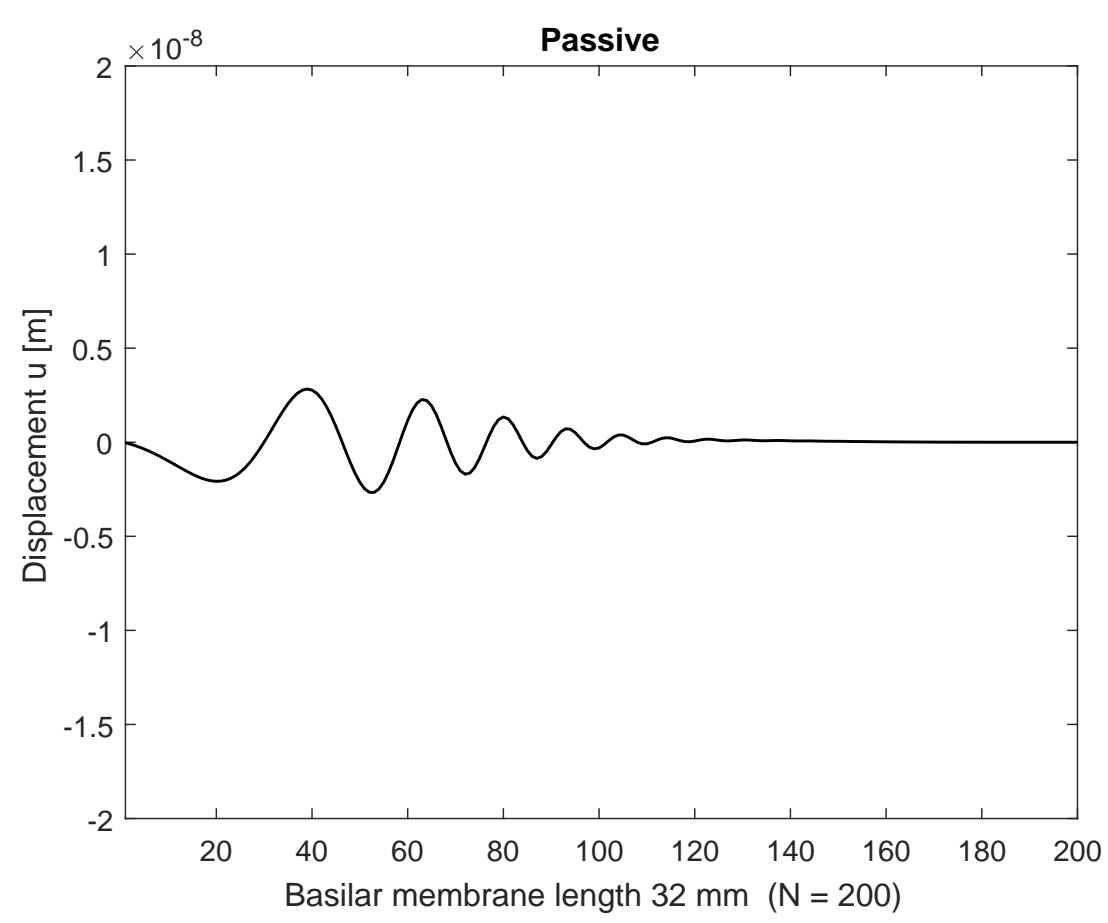

Figure 3. Displacements along the BM with stimulation frequency $2000 \mathrm{~Hz}$ at the base (passive), stimulation amplitude $\hat{u}=1 \mathrm{~nm}$. 
Initially the linear passive case with the constant Young's modulus $E_{y}$ is presented in space (Figure 3) after $100 \mathrm{~ms}$ and frequency (Figure 5) at a basal location $(6.4 \mathrm{~mm})$ or $n=40$ with $\hat{u}=1 \mathrm{~nm}$ stimulating displacement amplitude. The maximum displacement is located basally, and the output frequency remains the input frequency because of the linearity of the system. In case of parametric amplification by the time and space dependent Young's modulus, the amplitude of BM displacement increases to $18.8 \mathrm{~nm}$ and the place of maximum displacement shifts to the center of the BM length at $16 \mathrm{~mm}$ or $n=100$ (Figure 4). This increase corresponds to a BM displacement amplification of $15.53 \mathrm{~dB}$, evaluated by the fraction of the maximum displacement at half of the BM length with parametric amplification to the maximum at the more basal place $6.4 \mathrm{~mm}(n=40)$ in the passive case. Figure 6 shows the corresponding frequency spectrum for the active case. The examples considered so far would lead to an unlimited increase of displacements.

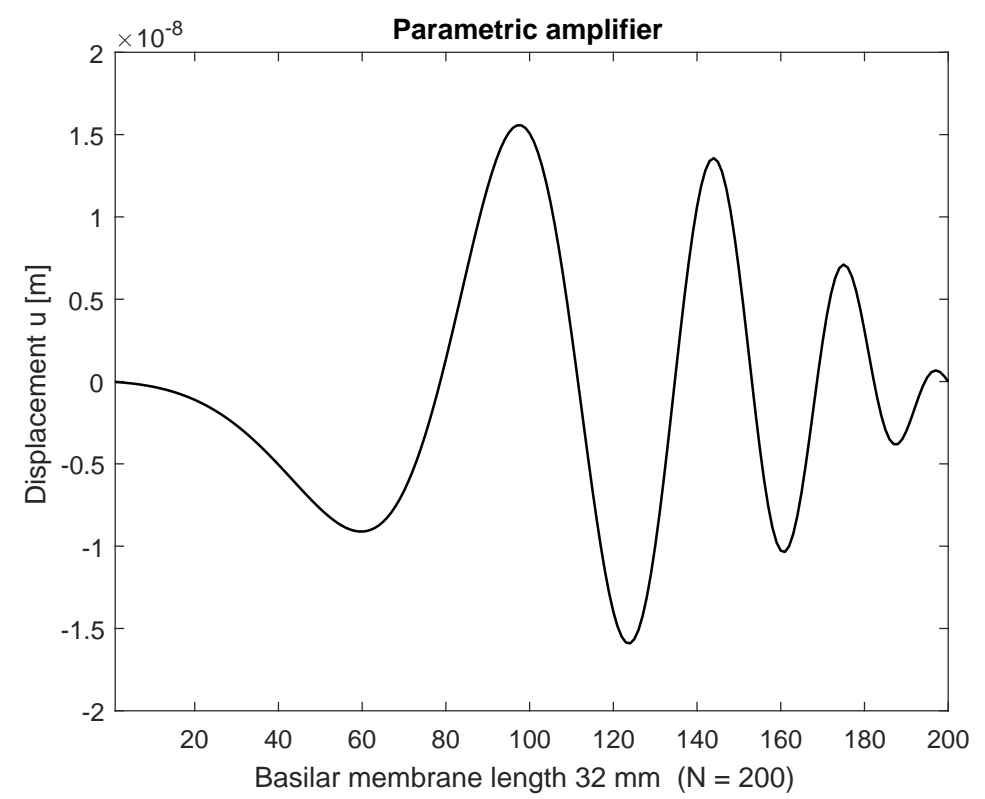

Figure 4. Displacements with stimulation frequency $2000 \mathrm{~Hz}$ (parametric amplified), stimulation amplitude $\hat{u}=1 \mathrm{~nm}$. Nonlinear distortions are generated by the OHC nonlinear stiffness function.

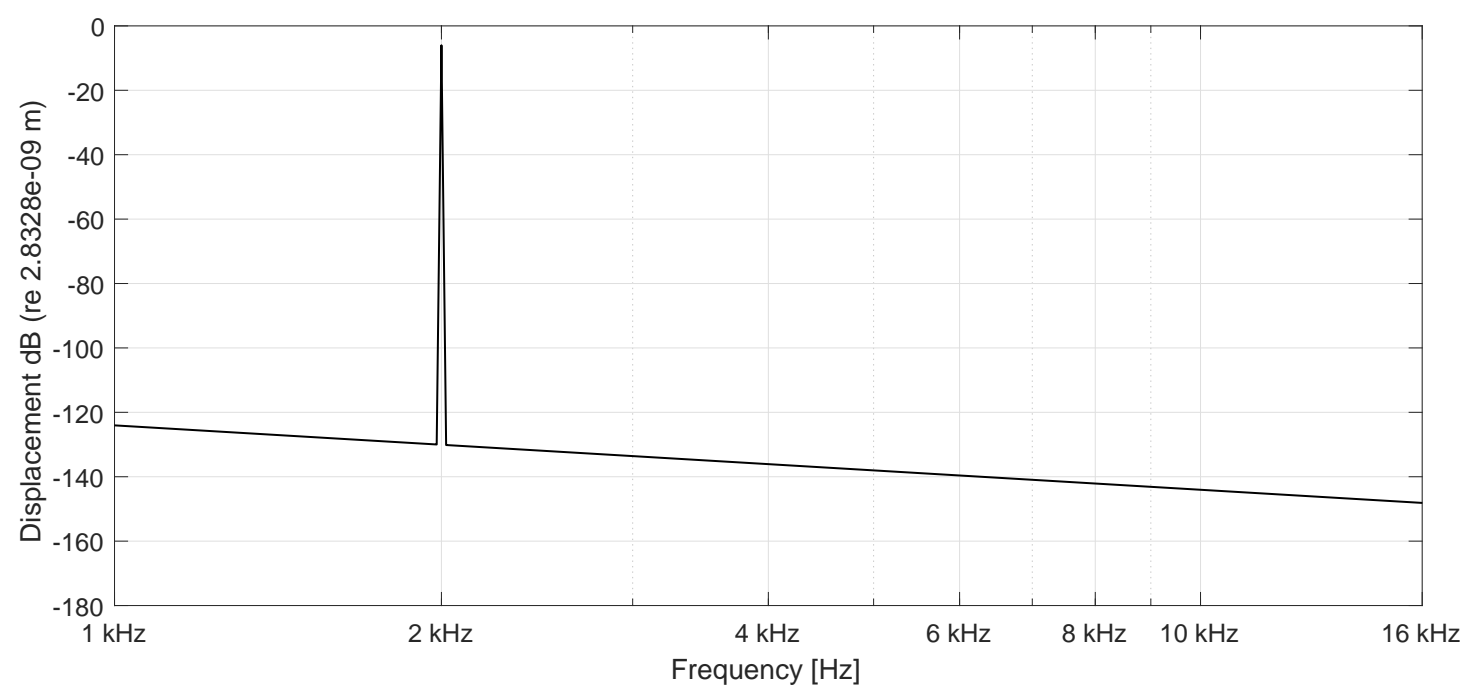

Figure 5. Spectrum of BM displacement at $6.4 \mathrm{~mm}(n=40)$ stimulation frequency $2000 \mathrm{~Hz}$ (passive), stimulation amplitude $\hat{u}=1 \mathrm{~nm}$. 


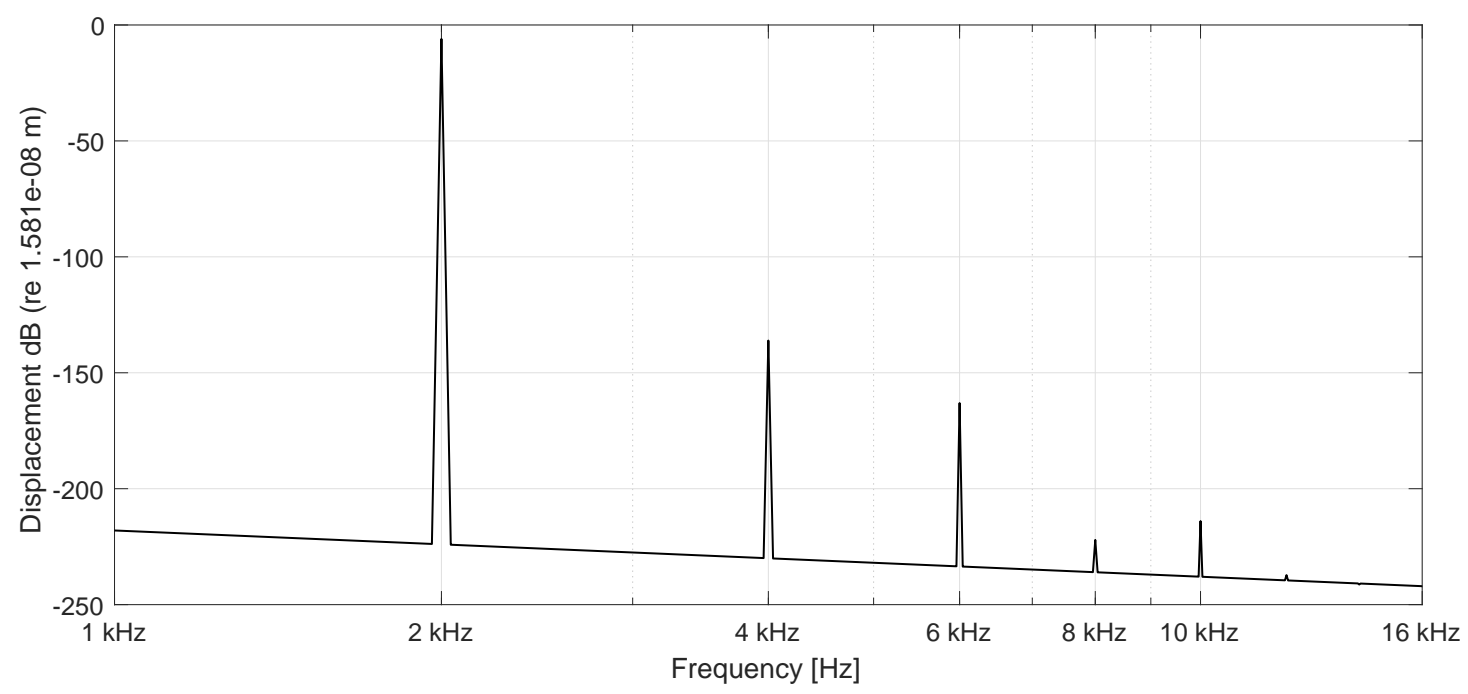

Figure 6. Spectrum of BM displacements at $16 \mathrm{~mm}(n=100)$ stimulation frequency $2000 \mathrm{~Hz}$ (active), stimulation amplitude $\hat{u}=1 \mathrm{~nm}$.

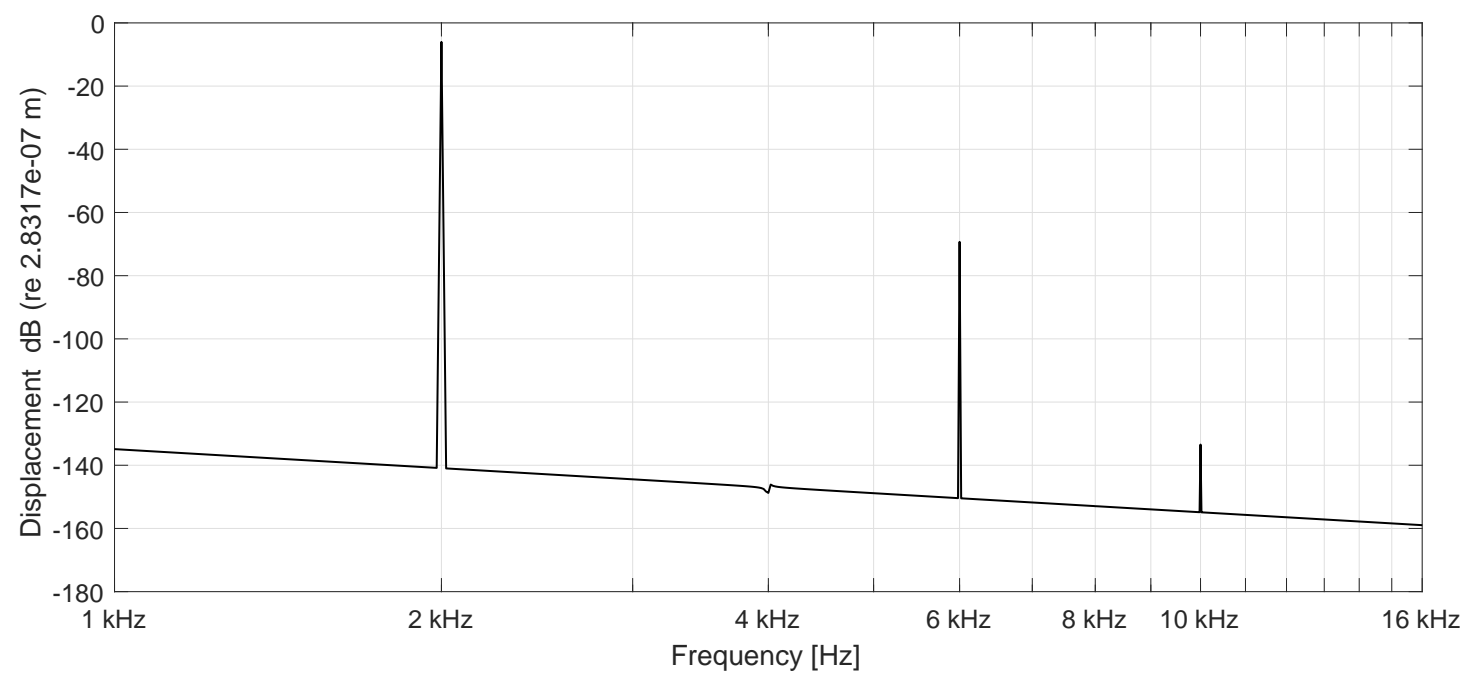

Figure 7. Spectrum of BM displacements at $6.4 \mathrm{~mm}(n=40)$ stimulation frequency $2000 \mathrm{~Hz}$ (passive), stimulation amplitude $\hat{u}=100 \mathrm{~nm}$. Nonlinear distortions are generated by the cubic nonlinearity of the string.

Therefore, the cubic term in Equations (2) and (3) representing the nonlinear increase of the restoring force with increasing displacement is included. The passive case in Figure 7 shows the spectrum of displacements at the base $(6.4 \mathrm{~mm})$ of the $\mathrm{BM}$ with a higher stimulating amplitude of $\hat{u}=100 \mathrm{~nm}$. The odd harmonics $\left(y^{2}+y^{4}+y^{6} \ldots\right) y$ are the distortion components of a string, limited in its vibration amplitude by a geometrical nonlinearity caused by an increased stiffness with increasing displacement amplitudes. Figure 8 presents displacements of the healthy cochlea with parametric amplification and an increase of displacements by $23.97 \mathrm{~dB}$ compared to the passive case. It even shows odd harmonics, caused by the nonlinear OHC stiffness and the saturating displacements induced by the stiffening of the nonlinear string (BM). 


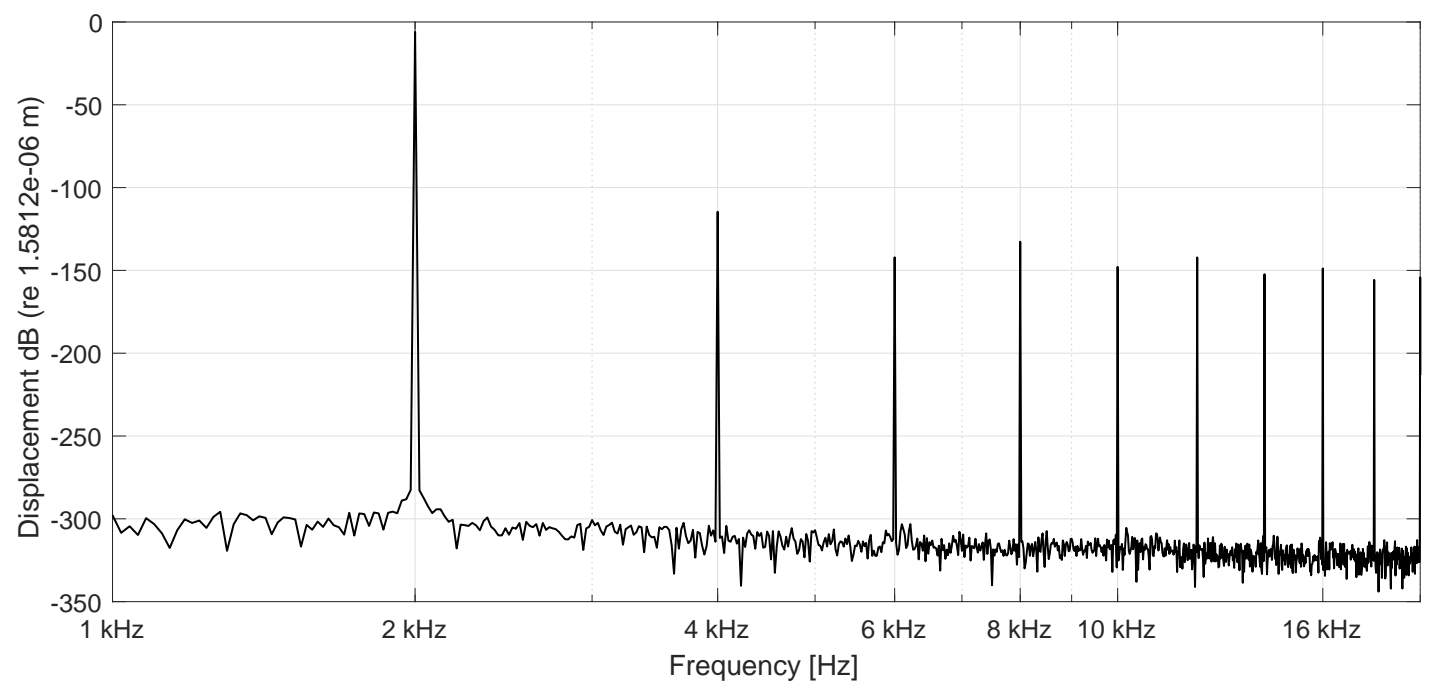

Figure 8. Spectrum of BM displacements at $16 \mathrm{~mm}(n=100)$ stimulation frequency $2000 \mathrm{~Hz}$ (active), stimulation amplitude $\hat{u}=100 \mathrm{~nm}$. Nonlinear distortions are generated by the $\mathrm{OHC}$ stiffness functions and the saturating cubic nonlinearity of the string.

\section{Discussion and Conclusions}

The BM represented as a string vibrates with the characteristics of a traveling wave, namely, the frequency to characteristic place transformation. A main result of this work is the dependence of this transformation by the time-varying Young's modulus, causing parametric amplification (PA). The numerical examples prove the effect of PA in a 1D mechanical-mathematical model of the cochlea. Therefore, the frequency to place-map is largely dependent on the PA demonstrated by the difference in Figures 3 and 4, which are supplied by input signals of identical amplitude and frequency. The amplification of $15.53 \mathrm{~dB}$ for the low level case; $24.0 \mathrm{~dB}$ for the high level case; and the place shift of maximum displacement along the BM with the same input frequency, corresponds to experimental data from the squirrel monkey [15]. The PA is achieved by changing the axial stiffness of $\mathrm{OHC}$ represented by the Young's modulus change $\Delta E_{y}$. In contrast to former approaches of PA applied to the cochlea [16], where the OHC contraction is in common mode with the input signal frequency and also constant in space, the stiffness is time and space dependent according to arbitrary and variable BM displacements. Another work includes the PA by outer hair cells, but reduces it to one location along the BM without considering traveling waves [17]. Therefore, the inclusion of a signal-dependent stiffness change along the space coordinate is a main advantage to former approaches. For higher input levels, geometric nonlinearities of the cells of the organ of Corti become relevant [18] and saturate the displacements leading to the limitation of BM displacements by geometrical nonlinearities. For simplicity, these saturation mechanisms are represented by a cubic nonlinearity of the transversal vibrations of a string neglecting bending stiffness and longitudinal deformations in the present work.

A comparison of the results with experimental data is merely possible fractionally. The fact of the frequency-dependent maximum BM displacements is fulfilled by displacements shown in Figure 3 (passive) and Figure 4 (active). They show the maximum displacements in the medial part of the string, or BM as it was measured by von Békésy in human temporal bones [19]. The shift of BM displacement amplitudes from the active (in vivo) case to the passive (dead) case is observable in Figures 3 and 4. The experimental validation of this progression is given by the data of Rhode et al. (1974). In Figure 3 of this work [15], the ratio of basilar membrane displacement/malleus displacement is presented log-scaled (amplitude and frequency). At the frequency $f_{\text {Rhode }}=6 \mathrm{kHz}$, which should correspond to the frequency $f=2 \mathrm{kHz}$ in this work, an amplitude decrease of $20 \mathrm{~dB}$ is found from the active in vivo case to the passive ( $1 \mathrm{~h}$ after death) case. This measurement was conducted at one place and it is impossible to measure BM displacements at many places simultaneously, as it is 
possible in numerical models of the organ. Nonetheless, the experiments confirm the place shift of the maximum BM displacement along the cochlea to the base (direction of the stapes) without the parametric amplification of OHC (passive case). Therefore, the in vivo activity shifts the maximum BM displacement to the apex (direction of the helicotrema), as can be seen in Figures 3 and 4 of this work.

Another comparison with experimental data could be conducted with measured otoacoustic emissions (OAE) e.g., distortion product otoacoustic emissions (DPOAE), applying two tones with a frequency ratio of e.g., $f_{2} / f_{1}=1.2$ and different levels of primary tones at the base of the string. However, a comparison with measured DPOAE requires a three-dimensional (3D) mechanical mathematical model of the cochlea because the generator of the DPOAE, which is the nonlinear stiffness of $\mathrm{OHC}$, leads to high frequency mechanical waves in the surrounding fluid. These waves interfere and are measurable with a sensor in the cochlea [20] or in the ear canal [21]. As the fluid is not modeled as a dimensional continuum yet, it is not possible to evaluate the interfering components and produce realistic signals to be comparable with measured OAE.

Nevertheless, it is shown that the inclusion of small stiffness changes of the BM enables a parametric amplification of acoustic signals caused by nonlinear distortions at low levels in a 1D model of cochlear mechanics. Though nonlinear cochlear preprocessing has been proposed before [22,23], these physical concepts did not include the PA and therefore the level dependent BM displacements did not show frequency-dependent shifts along the BM to be inferred from PA.

The PA causes nonlinear distortions, measurable as OAE propagating inside the cochlea which are emitted into the ear canal. An evaluation of OAE requires a 3D model of the cochlea to be presented in a future work. These simulations will include the 3D wave propagation in the fluid and the active BM with nonlinear OHC functions introduced by time and space variable Young's moduli of the solid or BM. This approach will prove the concept of PA and will enable the calculation of OAE, which are by-products of the cochlear amplifier, which has been evolved to increase the hearing sensitivity.

Funding: This research received no external funding.

Acknowledgments: All calculations were executed using MATLAB R2016b.

Conflicts of Interest: The author declares no conflict of interest.

\section{References}

1. Melde, F. Über die Erregung stehender Wellen eines fadenförmigen Körpers [On the excitation of standing waves on a string]. Annalen der Physik und Chemie (Ser. 2) 1859, 109, 193-215.

2. Cullen, A.L. Theory of the travelling-wave parametric amplifier. Proc. IEE-Part B 1959, 107, 101-107. [CrossRef]

3. Morse, P.M.; Ingard, K.U. Theoretical Acoustics; Princeton University Press: Princeton, NJ, USA, 1986.

4. Nayfeh, A.H.; Mook, D.T. Nonlinear Oscillations; Pure \& Applied Mathematics; John Wiley \& Sons: New York, NY, USA, 1979.

5. Cheng, X.; Blanchard, A.; Tan, C.A.; Lu, H.; Bergman, L.A.; McFarland, D.M.; Vakakis, A.F. Separation of traveling and standing waves in a finite dispersive string with partial or continuous viscoelastic foundation. J. Sound Vibr. 2017, 411, 193-209. [CrossRef]

6. Tan, C.A.; Yang, B.; Mote, C.D., Jr. More On the vibration of a translating string coupled to hydrodynamic bearings. J. Vib. Acoust. 1990, 112, 337-345. [CrossRef]

7. Huang, F.Y.; Mote, C.D., Jr. On the translating damping caused by a thin viscous fluid layer between a translating string and a translating rigid surface. J. Vib. Acoust. 1995, 181, 251-260. [CrossRef]

8. Böhnke, F.; Janssen, T.; Steinhoff, H.-J.; Zimmermann, P. Structural Cochlear Model including the Coupling of Basilar Membrane Fibers in the Longitudinal Direction. Il Valsalva 1989, 54 (Suppl. 1), 10-16.

9. Elliott, S.J. Wave propagation in a constrained fluid layer bounded by an elastic half-space and its relevance in cochlear micromechanics. J. Sound Vib. 2007, 305, 918-924. [CrossRef]

10. Böhnke, F.; Semmelbauer, S. Acoustic boundary layer attenuation in ducts with rigid and elastic walls applied to cochlear mechanics. J. Fluids Eng. 2017, 139, 101202. [CrossRef] 
11. Najafi, H.S.; Izadi, F. Comparison of two finite-difference methods for solving the damped wave equation. Int. J. Math. Eng. Sci. 2014, 3, 35-49.

12. Preyer, S.; Gummer, A.W. Nonlinearity of mechanoelectrical transduction of outer hair cells as the source of nonlinear basilar-membrane motion and loudness recruitment. Audiol. Neuro-Otol. 1996, 1, 3-11. [CrossRef]

13. He, D.Z.; Dallos, P. Somatic stiffness of cochlear outer hair cells is voltage-dependent. Proc. Natl. Acad. Sci. USA 1999, 96, 8223-8228. [CrossRef]

14. Deo, N.; Grosh, K. Two-State Model for Outer Hair Cell Stiffness. Biophys. J. 2004, 86, 3519-3528. [CrossRef] [PubMed]

15. Rhode, W.S.; Robles, L. Evidence from Mössbauer experiments for nonlinear vibration in the cochlea. J. Acoust. Soc. Am. 1974, 55, 588-596. [CrossRef] [PubMed]

16. Ko, W.; Stockie, J.M. An immersed boundary model of the cochlea with parametric forcing. SIAM J. Appl. Math. 2015, 75, 1065-1089. [CrossRef]

17. Kielczynski, P. Power amplification and selectivity in the cochlear amplifier. Arch. Acoust. 2013, 38, 83-92. [CrossRef]

18. Böhnke, F.; Arnold, W. Mechanics of the Organ of Corti Caused by Deiters Cells. IEEE Trans. Biomed. Eng. 1998, 45, 1227-1233. [CrossRef] [PubMed]

19. von Békésy, G. Zur Theorie des Hörens, Die Schwingungsform der Basilarmembran. Physikalische Zeitschrift 1928, 29, 793-810.

20. Dong, W. Simultaneous intracochlear pressure measurements from two cochlear locations: Propagation of distortion products in gerbil. J. Assoc. Res. Otolaryngol. 2017, 18, 209-225. [CrossRef]

21. Kemp, D.T. Evidence of mechanical nonlinearity and frequency selective wave amplification in the cochlea. Arch. Otorhinolaryngol. 1979, 224, 37-45. [CrossRef]

22. Zwicker, E. A hardware cochlear nonlinear preprocessing model with active feedback. J. Acoust. Soc. Am. 1986, 80, 146-153. [CrossRef]

23. Verhulst, S.; Dau, T.; Shera, C.A. Nonlinear time-domain cochlear model for transient stimulation and human otoacoustic emission. J. Acoust. Soc. Am. 2012, 132, 3842-3848. [CrossRef] [PubMed]

(C) 2019 by the authors. Licensee MDPI, Basel, Switzerland. This article is an open access article distributed under the terms and conditions of the Creative Commons Attribution (CC BY) license (http://creativecommons.org/licenses/by/4.0/). 